\title{
Comunicação
}

[Communication]

\section{Produção de enterotoxinas e da toxina da síndrome do choque tóxico por cepas de Staphylococcus aureus isoladas na mastite bovina}

[Production of enterotoxins and toxic shock syndrome toxin by Staphylococcus aureus strains isolated from bovine mastitis]

\author{
A. Nader Filho ${ }^{1 *}$, L.M. Ferreira ${ }^{2}$, L.A. Amaral ${ }^{1}$, O.D. Rossi Junior ${ }^{1}$, R.P. Oliveira ${ }^{1}$ \\ ${ }^{1}$ Faculdade de Ciências Agrárias e Veterinárias - UNESP \\ Via de Acesso Prof. Paulo Donato Castelane $\mathrm{s} / \mathrm{n}^{\circ}$ \\ 14884-900 - Jaboticabal, SP \\ ${ }^{2}$ Aluno de pós-graduação - FCAV-UNESP - Jaboticabal, SP
}

Staphylococcus aureus está envolvido em infecções intramamárias de fêmeas em lactação, sendo o principal agente causador da mastite em bovinos (Cardoso et al., 2000). Algumas cepas desse microrganismo podem produzir uma grande variedade de toxinas extracelulares e de fatores de virulência (Matsunaga et al., 1993), cuja presença no leite e seus derivados pode representar sério problema de saúde pública (Ichikawa et al., 1996).

Enterotoxinas estafilocócicas (SE) são os principais agentes de intoxicação de origem bacteriana no homem e têm sido relatadas em vários surtos de doenças transmissíveis por alimentos (Lamaita et al., 2005).

As toxinas são termoestáveis (Cliver, 1994) e o período de incubação é bastante curto, variando de 15 minutos a 6 horas após a ingestão do alimento contaminado (Carmo, 2001). Segundo Bergdoll (1989), uma mesma cepa de $S$. aureus pode produzir mais de um tipo de toxina, que em quantidades inferiores a $1 \mu \mathrm{g}$ podem desencadear os sintomas de intoxicação, representados, principalmente por vômitos e diarréias. Casos mais graves podem ocorrer entre os récemnascidos, idosos e pessoas acometidas por doenças crônicas imunossupressoras (Cliver, 1994).

Outro fator de virulência é representado pela toxina 1 da síndrome do choque tóxico (TSST-1)

Recebido em 23 de março 2006

Aceito em 3 de setembro de 2007

E-mail: nader@fcav.unesp.br sendo reconhecida como causadora febre, hipotensão, congestão de vários órgaos e choque letal (Bergdoll e Chesney, 1991).

Tendo em vista a importância dos S. aureus como agentes etiológicos da mastite bovina, considerando os riscos que o leite contaminado por estes microrganismos pode representar para a saúde pública, idealizou-se o presente trabalho com o objetivo de verificar a produção de SE e da TSST-1. Para tanto, foram utilizadas 72 cepas de $S$. aureus isoladas no leite de vacas com sinais de mastite ou que apresentavam positividade no California Mastitis Test, em 10 propriedades rurais do Estado de São Paulo.

A detecção das SE e da TSST1 foi realizada pela equipe do doutor Luiz Simeão do Carmo, do laboratório de enterotoxinas estafilocócicas da Fundação Ezequiel Dias, que utilizou o método de placa de sensibilidade ótima (optimumsensitivity plate method - OSP) descrito por Robbins et al. (1974).

Na Tab. 1 mostra-se que das 72 cepas de $S$. aureus estudadas, 66 (91,7\%) apresentaram produção de pelo menos um tipo de toxina, isoladamente ou em associação, evidenciando, portanto, elevado grau de toxigenicidade. Revela, também, que $12(16,7 \%)$ produziram apenas um tipo de toxina, enquanto $10(13,9 \%)$ produziram, simultaneamente, até quatro $\mathrm{SE}$, e que apenas seis $(8,3 \%)$ mostraram-se negativas na produção das toxinas SEA, SEB, SEC, SED e TSST-1. 
A Fig.1 apresenta a distribuição da produção das SE e da TSST-1 entre as 72 cepas de $S$. aureus estudadas, isoladamente, desprezando-se as associações. As toxinas SEA $(52,8 \%)$ e SEB $(52,8 \%)$ foram as mais produzidas, seguidas por SED (44,4\%), SEC (38,9\%) e TSST-1 (37,5\%).

Entre as toxinas identificadas, houve predomínio da SEA e SEB em relação às outras toxinas estafilocócicas. Este resultado difere dos obtidos por Lopes et al. (1990), em São Paulo, por Cardoso et al. (2000), em Minas Gerais, e por Sá et al. (2004), em São Paulo, que verificaram maior ocorrência da SEA e SEC, TSST-1 e SED e da SEC e SED, respectivamente. Cardoso et al. (2000) acreditam que as diferenças observadas nas freqüências e nos tipos de toxinas extracelulares talvez possam ser atribuídas aos métodos de detecção das toxinas e as características das cepas de $S$. aureus isoladas de diversas origens.

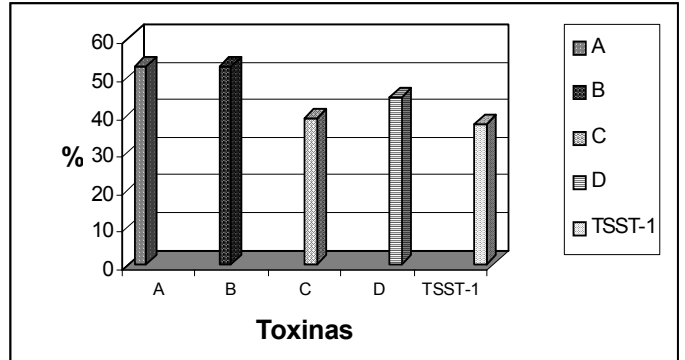

Figura 1. Distribuição da produção das toxinas estafilocócicas em 72 cepas de $S$. aureus isoladas em casos de mastite bovina diagnosticados em 10 propriedades rurais do Estado de São Paulo, 2002.

Com relação à TSST-1, deve-se ressaltar que essa toxina ganhou notoriedade por ser detectada com freqüência nos casos de mastite bovina, ovina e caprina (Jones e Wieneke, 1986; Matsunaga et al., 1993; Bezek e Hull, 1995). O primeiro relato de detecção da TSST-1 produzida por $S$. aureus de origem animal no Brasil é atribuído a Cardoso et al. (2000), que verificaram a produção dessa toxina em $47,2 \%$ das cepas isoladas de casos de mastite bovina em Minas Gerais. A freqüência $(37,5 \%)$ de isolamento de cepas de $S$. aureus produtoras de TSST-1 observada neste trabalho (Fig. 1) foi menor que a obtida por Cardoso et al. (2000). Sá et al (2004) não identificaram a ocorrência de cepas produtoras dessa toxina nos casos de mastite estudados na região de Botucatu/SP.
Tabela 1. Produção de enterotoxinas estafilocócicas (SE) e de toxina da síndrome do choque tóxico (TSST-1) pelas cepas de S. aureus isoladas em casos de mastite bovina diagnosticados em 10 propriedades rurais do Estado de São Paulo, 2002

\begin{tabular}{lcr}
$\begin{array}{l}\text { Enterotoxina estafilocócica } \\
\text { (SE) }\end{array}$ & $\begin{array}{r}\text { Número } \\
\text { de cepas }\end{array}$ & $\%$ \\
\hline SEA & 2 & 2,8 \\
SEB & 5 & 6,9 \\
SEC & 5 & 6,9 \\
SEA + SEB & 1 & 1,4 \\
SEB + SEC & 17 & 23,6 \\
SEA + TSST-1 & 3 & 4,2 \\
SEA + SEB + SED & 4 & 5,6 \\
SEA + SEC + SED & 2 & 2,8 \\
SEA + SED + TSST-1 & 16 & 22,2 \\
SEB + SEC + TSST-1 & 1 & 1,4 \\
SEA + SEB + SEC + SED & 3 & 4,2 \\
SEA + SEB + SED + TSST-1 & 7 & 9,7 \\
Nenhuma & 6 & 8,3 \\
Total & 72 & 100,0 \\
\hline
\end{tabular}

A mesma cepa de $S$. aureus pode produzir mais de um tipo de enterotoxina, como observado neste trabalho (Tab. 1). Achados semelhantes foram relatados por Cardoso et al. (2000) em Minas Gerais, a exemplo dos observados por alguns autores em outras regiões do mundo (Jones e Wieneke, 1986; Matsunaga et al.,1993; Ichikawa et al.,1996; Takeuchi et al., 1998). Embora Soares et al. (1997) tenham assinalado que a co-produção de diferentes tipos de toxinas possa sugerir maior toxigenicidade dessas cepas na patogenia das infecções produzidas por esses microrganismos, e Refai et al. (1988) tenham demonstrado a relação existente entre a atividade enzimática específica, a enterotoxigenicidade e a resistência dos $S$. aureus a vários antibióticos, principalmente naquelas produtoras de mais de um tipo de enterotoxina, os autores citados não discutiram a importância desse resultado.

Embora sejam variáveis as freqüências de isolamento de cepas de $S$. aureus em amostras de leite de vacas com mastite, esse agente pode ser considerado mundialmente como o de maior significado na etiologia dessa enfermidade (Sá et al., 2004). Este fato, aliado ao elevado índice de cepas enterotoxigênicas, como o verificado nesta investigação, deve merecer especial atenção dos órgãos oficiais de inspeção e de vigilância sanitária, uma vez que pode representar sério 
risco potencial para a saúde pública (Lamaita et al., 2005).

Apesar da simples presença das cepas de $S$. aureus enterotoxigênicas não implicar necessariamente na ocorrência de casos intoxicações, sabe-se que o leite constitui em excelente substrato para a proliferação desses microrganismos e que a temperatura da glândula mamária é ideal para a produção de enterotoxinas em concentrações suficientes para causar a doença no homem.
Os achados deste trabalho adquirem uma importância ainda maior principalmente se considerar a termoestabilidade das enterotoxinas estafilocócicas pré-formadas e a conseqüente possibilidade da sua persistência tanto no leite pasteurizado como em seus derivados.

Palavras-chave: bovino, mastite, Staphylococcus aureus, enterotoxinas

\begin{abstract}
A total of 72 strains of Staphylococcus aureus were examined for the production of staphylococcal enterotoxins (SE) A, B, C, D and toxic shock syndrome toxin (TSST-1). The strains were isolated from milk samples from cows with mastitis in dairy herds of São Paulo State, Brazil. Off 72 isolates, 38 (52.8\%) produced SEA, 38 (52.8\%) SEB, 32 (44.4\%) SED, 28 (38.9\%) SEC and 27 (37.5\%) TSST-1. From the 72 strains, 66 (91.7\%) produced, at least, one or more toxin, including TSST-1.
\end{abstract}

Keywords: bovine, mastitis, Staphylococcus aureus, enterotoxins, TSST-1

\section{REFERÊNCIAS BIBLIOGRÁFICAS}

BERGDOLL, M.S. Staphylococcus aureus. In: Foodborne bacterial pathogens. New York: Marcel Dekker, 1989. p.463523.

BERGDOLL, M.S.; CHESNEY, P.J. (Eds). Toxic shock syndorme. Boston: CRC, 1991. 235p.

BEZEK, D.M.; HULL, B.L. Peracute gangrenous mastitis and cheilitis associated with enterotoxin-secreting Staphylococcus aureus in a goat. Can. Vet. J., v.36, p.106107, 1995.

CARDOSO, H.F.T.; CARMO, L.S.; SILVA, N. Detecção da toxina-1 da síndrome do choque tóxico em amostras de Staphylococcus aureus isoladas de mastite bovina. Arq. Bras. Med. Vet. Zootec., v.52, p.7-10, 2000.

CARMO, L.S. Produção e purificação em grande escala das enterotoxinas estafilocócicas SEA, SEB, SEC , SED e TSST-1 para uso em ensaios imuno-enzimáticos. 2001. 254f. Tese (Doutorado) - Instituto de Ciências Biológicas, Universidade Federal de Minas Gerais, Belo Horizonte.

CLIVER, D.O. (Ed). Foodborne disease handbook: diseases caused by bacteria. New York: Marcel Dekker, 1994. 613p.

ICHIKAWA, M.; ICHIKAWA, T.; MIZOMOTO, T. Productivity of enterotoxins and toxic shock syndome toxin1, and coagulase type of Staphylococcus aureus strains isolated from bovines and humans in the same district. Anim. Sci. Technol., v.67, p.780-786, 1996

JONES, T.O.; WIENEKE, A.A. Staphylococcal toxic shock syndrome. Vet. Rec., v.119, p.435, 1986.

LAMAITA, H.C.; CERQUEIRA, M.M.O.P.; CARMO, L.S. et al. Staphylococcus sp. counting and detection of staphylococcal enterotoxins and toxic shock toxin syndrome from cooled raw milk. Arq. Bras. Med. Vet. Zootec., v.57, p.702-709, 2005.

LOPES, C.A.M.; MORENO, G.; CURI, P.R. et al. Characteristics of Staphylococcus aureus from subclinical bovine mastitis in Brasil. Br. Vet. J., v.146, p.443-446, 1990.

MATSUNAGA, T.; KAMATA, S.; KIKIICHI, N. et al. Characteristics of Staphylococcus aureus isolated from peracute, acute and chronic bovine mastitis. J. Med. Sci., v.55, p.297-300, 1993.

QUINN, P.J.; CARTER, M.E.; MARKEY, B. et al. (Eds). Clinical veterinary microbiology, London: Wolfe, 1994. 648 p.

REFAI, M.; NIAZI, Z.M., YOUSSEF, S.A.H. et al. Correlation between antibiotic-resistence, enterotoxigenicity and enzymatic activities of Staphylococcus aureus recovered from foods. Vet. Med. J., v.36, p.107-119, 1988.

ROBBINS, R.; GOULD, S.; BERGDOLL, M.S. Detecting the enterogenicity of Staphylococcus aureus strains. Appl. Microbiol., v.28, p.946-950, 1974.

SÁ, M.E.P.; CUNHA, M.S.R.S.; ELIAS, A.O. et al. Importância do Staphylococcus aureus nas mastites subclínicas: pesquisa de enterotoxinas e toxina do choque tóxico, e a relação com a contagem de células somáticas. Braz. J. Vet. Res. Anim. Sci., v.41, p.321-326, 2004

SOARES, M.J.S.; TOKUMARU-MIYAZAKI, N.H.; NOLETO, A.L.S. Enterotoxin production by Staphylococcus aureus clones and detection of Brazilian epidemic MRSA clone (III::B:A) among isolates from food handlers. J. Med. Microbiol., v.46, p.214-221, 1997.

TAKEUCHI, S.; ISHIGURO, K.; IKEGAMI, M. et al. Production of toxic shock syndrome toxin by Staphylococcus aureus isolated from mastitic cow's milk and farm bulk milk. Vet. Microbiol., v.59, p.251-258, 1998. 